\title{
Hepatitis C infection, related services, and barriers to HCV treatment among drug users in methadone maintenance treatment (MMT) clinics in Shanghai, China
}

Zhi-Bin $\mathrm{Li}^{6+}$, Lei Zhang ${ }^{1 \dagger}$, Jun Wang ${ }^{2}$, Le-Ping Huang ${ }^{3}$, Zhi-Rong Zhou ${ }^{4}$, Yi-Ning Cao ${ }^{5}$, Min Zhao ${ }^{1,7,8^{*}}$ and Jiang Du ${ }^{1 *}$

\begin{abstract}
Background: The purpose of this study was to document the prevalence of hepatitis $C$ among MMT patients, hepatitis $\mathrm{C}$ virus $(\mathrm{HCV})$ knowledge of patients and MMT staff members, and the barriers preventing them from receiving or delivering HCV-related services in MMT clinics of China.
\end{abstract}

Methods: Data were collected from 240 MMT patients and 58 staff members in Shanghai MMT clinics. Structured questionnaires (HCV Knowledge Scale and Alcohol Use Disorders Identification Test) and several self-developed questionnaires were used to assess (1) patient and staff HCV knowledge, (2) attitudes toward HCV-related services in MMT clinics, and (3) what type of HCV-related services the staff members have provided in their routine work. The HCV test results were based on the patients' medical records.

Results: The HCV seropositive rate was high (70\%), and both patients and staff had limited HCV knowledge. The mean score of patient HCV knowledge was 6.8 out of 20 (SD = 3.7), whereas the mean score of staff HCV knowledge was 10.9 out of 20 (SD = 3.1). For HCV-positive patients, only $13.7 \%$ had accessed HCV medical treatment. Barriers included the cost of medical treatment, lack of HCV knowledge, lack of professional training for patients to receive HCV-related services from individuals or MMT clinics, and lack of an adequate policy-making system.

Conclusions: HCV infection remains an important problem among MMT patients in China. Barriers to HCV-related services are attributable to individual, clinical, and policy-related factors. This study may provide evidence-based information for future work to optimize the resources of MMT clinics.

Trial registration: ClinicalTrials.gov NCT01647191. Registered 17 April 2012.

Keywords: Hepatitis C, Methadone maintenance treatment, Heroin dependent, China

\section{Background}

Hepatitis $\mathrm{C}$ virus (HCV) infection is a serious public health problem worldwide. The World Health Organization (WHO) has estimated that 185 million people are chronically infected with HCV globally, and at least $3.7 \%$ of East Asian people are HCV positive [1]. With an estimated population of 1.3 billion, the number of HCV-infected people in China could exceed 48

\footnotetext{
* Correspondence: drminzhao@gmail.com; dujiangdou@163.com

${ }^{\dagger}$ Equal contributors

${ }^{1}$ Shanghai Mental Health Center, Shanghai Jiao Tong University School of Medicine, Shanghai, China

Full list of author information is available at the end of the article
}

million. $\mathrm{HCV}$ infection has become one of the most important causes of chronic hepatitis, cirrhosis, and hepatocellular carcinoma in China [2].

High-risk factors for HCV infection include injection drug use (IDU) and transfusion of blood products. Since the establishment of a security system for blood donors in China, IDU has been the predominant mode of HCV transmission. By the end of 2014, approximately 2.96 million drug users were documented in China [3]. HCV infection has become a major health burden in China with rapid transmission, a long duration of clinical progression, and limited access of infected addicts to routine treatment. 
Despite the high rate of $\mathrm{HCV}$ infection among IDUs, the majority of drug users opt out of HCV services, and few engage in antiviral treatment for $\mathrm{HCV}$ compared to non-drug users [4-6]. To address the escalating HCV problem in IDU, the Chinese government conducts routine $\mathrm{HCV}$ testing in methadone maintenance treatment (MMT) clinics and provides $\mathrm{HCV}$-related training to MMT staff by the National MMT Training Center. However, this is not a sufficient training. Based on our previous study, drug users in MMT clinics had limited HCV knowledge, and most of them did not know their HCV status [7, 8].

Drug abuse treatment programs have been defined as a good platform to deliver $\mathrm{HCV} / \mathrm{HIV}$-related interventions or medical services. As a strategy to solve the problems of opiate abuse and HIV, the Chinese government established MMT clinics throughout the country beginning in 2004. By the end of 2011, there were 738 MMT clinics in the entire country, and 300,000 heroin users benefited from this service [9]. MMT clinics have been effective in preventing the spread of HIV/AIDS $[10,11]$. Given the same transmission route and the Chinese situation, incorporating $\mathrm{HCV}$ prevention/intervention strategies into the MMT setting could be performed more fully and effectively to prevent $\mathrm{HCV}$ related consequences and increase medical uptake.

However, no study has yet reported on HCV-related services and barriers for patients to receive $\mathrm{HCV}$ treatment among drug users in China. Therefore, the first step is to obtain information about (1) patient and staff knowledge, (2) attitudes toward HCV-related services in MMT clinics, and (3) what type of HCV-related service the staff have provided in their routine work. Therefore, we conducted this study (1) to document the prevalence of hepatitis $C$ among MMT patients, (2) to explore $\mathrm{HCV}$ knowledge and attitudes toward HCV services among patients and MMT staff, and (3) to investigate the HCV-related services provided by treatment staff and identify the barriers that prevent patients and treatment staff from using or providing related services in the clinics.

\section{Methods}

\section{Study participants}

MMT patients

There were 14 MMT clinics in Shanghai located in urban areas. Since the final aim of the proposal was to evaluate the effectiveness of $\mathrm{HCV} / \mathrm{HIV}$ comprehensive interventions among drug users in MMT clinics, we defined any MMT clinic with more than 100 patients as meeting the inclusion criteria. There were nine MMT clinics that met the criteria, and four of them were randomly selected. At each selected clinic, the study was explained to potential participants during the recruitment process. For those who decided to participate in this study, detailed informed consent procedures outlined the nature of participation, risks, and benefits.

The patient eligibility criteria included opiate dependence (according to the criteria established by the Diagnostic and Statistical Manual IV, DSM-IV), at least 20 years of age or older, and being local residents. Sixty patients were randomly selected at each clinic for a total of 240 patients. All participants provided informed consent and were paid for their time.

The study participants were asked to respond to questions involving their socio-demographics, risk behaviors, and attitudes towards HCV-related services. They also provided consent to share clinical records and medical test results including drug use and $\mathrm{HAV}, \mathrm{HBV}$, and $\mathrm{HCV}$. The research staff provided support for participants who may have had challenges with comprehending study procedures or questionnaires.

\section{MMT staff}

Drug abuse treatment staff who currently work at Shanghai MMT clinics were invited to participate in this survey via flyers posted in their offices and mailed to their institutes. To be eligible, staff had to have been working in the MMT program for at least 1 year. There were no other screening criteria for service providers. A total of 58 MMT staff were invited from 14 MMT clinics in Shanghai.

The research protocol was approved by the Ethics Committee of the Shanghai Mental Health Center, and each subject signed the informed consent form approved by IRB at the Shanghai Mental Health Center.

\section{Instruments and measures}

Demographics for both patients and MMT staff and drug use history for patients were collected via a selfdeveloped questionnaire.

\section{Risk behaviors survey}

An abbreviated version of the risk behaviors assessment developed by the National Institute on Drug Abuse (NIDA) was used; it is used to measure the risk behaviors related to HIV and HCV. Risk behaviors in the areas of drug use and sex in the previous 30 days were measured. Reliability and validity assessments of the risk behaviors survey (RBS) support its adequacy as a research tool for populations of drug users [12-14].

\section{HCV knowledge questionnaire}

$\mathrm{HCV}$ knowledge questionnaire includes 20 items concerning $\mathrm{HCV}$ transmission risk and risk behaviors, $\mathrm{HCV}$ diagnosis and disease progression, current $\mathrm{HCV}$ treatment options, and treatment outcomes. One point is given for each correct answer with a possible score 
ranging from 0 to 20 . The instrument was translated into Chinese by one psychiatrist and back-translated by another psychiatrist, and it has been used in other studies with the same background [15-17].

\section{The Alcohol Use Disorder Identification Test}

The Alcohol Use Disorder Identification Test (AUDIT) was developed by the WHO to identify hazardous and harmful alcohol use and dependence, and it was specifically designed for international use [18]. This questionnaire consists of ten items to measure the quantity and frequency of alcohol use, possible dependence symptoms, and recent and lifetime problems associated with alcohol use. AUDIT was translated and validated in Chinese [19]. Alcohol consumption is an important factor in $\mathrm{HCV}$ infection prevention and treatment. Alcohol use may increase the risk of engaging in unprotected sex behaviors but can also exacerbate steatosis.

\section{HCV-related services survey (for patients)}

Perceptions of patients regarding $\mathrm{HCV}$ services were evaluated. The patients were asked to respond to a series of questions concerning $\mathrm{HCV}$-related education, $\mathrm{HCV} /$ HIV testing, and medical support services in their current treatment programs. They were asked to indicate the extent on a scale from 1 (do not agree at all) to 10 (completely agree) concerning the reason for using these services. Some of questions required a "Yes" or "No" response.

\section{HCV-related services survey (for MMT staff)}

A self-developed questionnaire was used to collect information of $\mathrm{HCV}$-related services provided by their treatment programs. It included $\mathrm{HCV}$-related training, $\mathrm{HCV}$ testing, and HCV medical treatment support. All questions had "Yes" or "No" as responses.

\section{Beck Depression Inventory}

Depressive symptoms were screened with the Chinese version of the 21-item Beck Depression Inventory. Each item is scored from 0 to 3 with a maximum score of 63 [20]. In this study, Beck Depression Inventory (BDI) was used to investigate the depression situation among MMT patients.

$\mathrm{HCV}$ tests are part of the routine procedure in MMT clinics in China. HCV serostatus was tested by enzymelinked immunosorbent assay (ELISA) in there. We obtained HIV, HAV, HBV, and HCV test results from the MMT clinic records with the permission of the participants and the clinic.

\section{Statistical analyses}

Statistical Product and Service Solutions 20.0 (SPSS 20.0) was used to conduct the statistical analyses.
Continuous data are presented as the mean and SD. Between-group differences for continuous variables were evaluated using Student's $t$ test. Between-group comparisons for categorical variables were performed using the chi-square test or Fisher's exact test when necessary.

\section{Results}

\section{Characteristics of the participants and hepatitis}

\section{prevalence}

The demographics of the participants and prevalence of hepatitis are summarized in Table 1 . Overall, $79.6 \%$ of the participants were male. The mean age was 42.0 (8.6) years, and nearly half of the participants were married or living with partners. Over half $(52.1 \%)$ had a middle school degree or less. There were no significant differences between $\mathrm{HCV}$-positive and $\mathrm{HCV}$-negative patients in those demographic characteristics.

A total of $70 \%$ of patients tested were positive for $\mathrm{HCV}$. Among those tested positive, 38.1\% reported their HCV status as negative or unknown. For HCV-negative patients, $16.7 \%$ reported that their test results were positive. Additionally, $8.3 \%$ of the participants were HBV positive. Participants who were $\mathrm{HCV}$ negative were

Table 1 Demographic and hepatitis prevalence by HCV status

\begin{tabular}{|c|c|c|c|}
\hline & $\begin{array}{l}\text { Total } \\
(n=240)\end{array}$ & $\begin{array}{l}\text { HCV positive } \\
(n=168)\end{array}$ & $\begin{array}{l}\text { HCV negative } \\
(n=72)\end{array}$ \\
\hline \multicolumn{4}{|l|}{ Gender, $n(\%)$} \\
\hline Males & $191(79.6)$ & $132(78.6)$ & $59(81.9)$ \\
\hline Females & $49(20.4)$ & $36(21.4)$ & $13(18.1)$ \\
\hline Age, mean (SD) & $42.0(8.6)$ & $41.2(8.5)$ & $43.8(8.7)$ \\
\hline \multicolumn{4}{|l|}{ Age, $n(\%)$} \\
\hline $21-35$ & $70(29.3)$ & $56(33.3)$ & $14(19.7)$ \\
\hline $36-50$ & $127(53.1)$ & $88(52.4)$ & $39(54.9)$ \\
\hline $51-61$ & $42(17.6)$ & $24(14.3)$ & $18(25.3)$ \\
\hline \multicolumn{4}{|l|}{ Marital status, $n(\%)$} \\
\hline Never married/single & $72(30.0)$ & $52(30.9)$ & $20(27.8)$ \\
\hline Married/living together & 115(47.9) & $85(50.6)$ & $30(41.7)$ \\
\hline Divorced/separated & $53(22.1)$ & $31(18.5)$ & $22(30.6)$ \\
\hline \multicolumn{4}{|l|}{ Education, $n$ (\%) } \\
\hline Middle school or less & $125(52.1)$ & $88(52.4)$ & $37(51.4)$ \\
\hline High school or higher & $115(47.9)$ & $80(47.6)$ & $35(48.6)$ \\
\hline \multicolumn{4}{|c|}{ Self-reported HCV test results, $n(\%)$} \\
\hline No & $84(35.0)$ & $39(23.2)$ & $45(62.5)$ \\
\hline Yes & $116(48.3)$ & $104(61.9)$ & $12(16.7)$ \\
\hline Unknown & $40(16.7)$ & $25(14.9)$ & $15(20.8)$ \\
\hline HAV (lgG) positive, $n(\%)$ & $104(43.7)$ & $76(45.5)$ & $28(39.4)$ \\
\hline HBV positive, $n(\%)^{*}$ & $20(8.3)$ & $10(5.9)$ & $10(13.9)$ \\
\hline HIV & 0 & 0 & 0 \\
\hline
\end{tabular}

${ }^{*} p<0.05$ 
significantly more likely to be infected with HBV compared to those who were $\mathrm{HCV}$ positive $\left(\chi^{2}=4.156\right.$, $p=0.041)$. No participants were infected with HIV.

\section{HCV knowledge and risk factors}

The 240 participants answered an average of 6.8 out of 20 items correctly in the HCV knowledge assessment, which demonstrated a poor $\mathrm{HCV}$ knowledge level among this population (Table 2). There were no significant differences in HCV knowledge between the $\mathrm{HCV}$-positive and HCV-negative groups. Regarding the drug use, injection, and sexual risk behavior history, the average period of drug use was 10.9 (6.7) years. HCV-positive patients had used drugs for a mean of 11.4 (7.2) years, which was significantly longer than the 10.0 (5.3) years of drug use reported by HCV-negative patients $(Z=1.674, p=0.047)$. Few (2.6\%) patients reported injection drug use in the past 30 days, and no one reported sharing injection needles. Almost $10 \%(9.6 \%)$ of the patients reported multiple sexual partners in past 30 days. More HCVnegative patients reported rarely or never using condoms with their casual or commercial sex partners compared to $\mathrm{HCV}$-positive patients.

In this study, $15 \%$ of patients reported drinking alcohol during the last year, and $8.3 \%$ met the criteria of harmful drinking. HCV-positive patients displayed more harmful drinking behavior than HCV-negative patients, but the difference was not significant.
Overall, three quarters of the patients had a selfreported poor health situation, and $45.4 \%$ had been depressed in the past 6 months. In total, $44.9 \%$ of the participants experienced mild depression or more severe depression symptoms in the last week according to the $\mathrm{BDI}$ and $16.3 \%$ expressed suicide notions. HCV-positive participants were more likely to have suicide notions compared to $\mathrm{HCV}$-negative participants.

\section{Patients' perceptions of HCV-related services}

When asked the rate of their needs for HCV education and medical support, the patients assigned scores of 6.0 (3.1) and 7.1 (2.9), respectively. For HCV testing services, $82.3 \%$ of the participants admitted that an $\mathrm{HCV}$ test is necessary to receive the treatment. Table 3 describes the participants' responses about barriers that prevent them from using HCV services. Above all, the patients reported that they really appreciated the HCV services provided in current treatment programs. However, only $13.7 \%$ of $\mathrm{HCV}$-positive patients accessed $\mathrm{HCV}$ medical treatment based on their self-report.

\section{MMT staff characteristics and HCV-related services provided in their clinics}

MMT staff knowledge of $\mathrm{HCV}$ was poor, with an average score of 10.9 (3.1). The most common education services provided in MMT clinics are individual counseling and booklets. In total, 39.7 and $35.6 \%$ of staff reported providing education services via group

Table 2 HCV knowledge and risk behaviors by HCV status

\begin{tabular}{|c|c|c|c|}
\hline & $\begin{array}{l}\text { Total } \\
(n=240)\end{array}$ & $\begin{array}{l}\text { HCV positive } \\
(n=168)\end{array}$ & $\begin{array}{l}\text { HCV negative } \\
(n=72)\end{array}$ \\
\hline HCV knowledge, mean (SD) & $6.8(3.7)$ & $7.0(3.5)$ & $6.4(3.8)$ \\
\hline Time of drug use (year), mean (SD)* & $10.9(6.7)$ & $11.4(7.2)$ & $10.0(5.3)$ \\
\hline Injection in past 30 days, $n$ (\%) & $6(2.6)$ & $6(3.8)$ & $0(0.0)$ \\
\hline Shared needles in past 30 days, $n(\%)$ & 0 & 0 & 0 \\
\hline More than one sexual partner in past 30 days, $n(\%)$ & $10(9.6)$ & $7(9.5)$ & $3(9.6)$ \\
\hline \multicolumn{4}{|l|}{ Not using condoms when having sex, $n(\%)$} \\
\hline With primary sex partner & $68(66.0)$ & $47(63.5)$ & $21(72.4)$ \\
\hline With casual sex partner & $14(14.0)$ & $9(12.3)$ & $5(18.5)$ \\
\hline With commercial sex partner & $12(12.0)$ & $6(8.3)$ & $6(21.4)$ \\
\hline Harmful drinking, $n(\%)$ & $20(8.3)$ & $15(8.9)$ & $5(6.9)$ \\
\hline Current health is poor by self-report, $n(\%)$ & $181(75.7)$ & $131(78.5)$ & $50(69.5)$ \\
\hline Depression symptoms in past 6 months, $n(\%)$ & $109(45.4)$ & $79(47.0)$ & $30(41.7)$ \\
\hline Suicide ideation in past 6 months, $n$ (\%) & $39(16.3)$ & $30(17.9)$ & $9(12.5)$ \\
\hline $\mathrm{BDI}>15, n(\%)$ & $107(44.9)$ & $72(43.4)$ & $35(48.6)$ \\
\hline
\end{tabular}

Note 1: Individuals who did not answer the sexual risk questions were dropped from the analysis. Thus, among the 240 participants, 104 were not included in the more than one sexual partner in past 30 days question, 103 were not included in the condom use with primary sex partner question, 100 were not included in the condom use with casual sex partner question, and 100 were not included in the condom use with commercial sex partner question

Note 2: Harmful drinking is defined by an AUDIT score of 7 or more

${ }^{*} p<0.05$ 
Table 3 Patients' perception of HCV-related services in their treatment programs

\begin{tabular}{|c|c|c|c|}
\hline & $\begin{array}{l}\text { Total } \\
(n=240)\end{array}$ & $\begin{array}{l}\text { HCV positive } \\
(n=168)\end{array}$ & $\begin{array}{l}\text { HCV negative } \\
(n=72)\end{array}$ \\
\hline Need HCV-related training when receiving treatment, mean (SD) & $6.0(3.1)$ & $6.1(3.2)$ & $5.9(3.2)$ \\
\hline Need HCV testing when receiving treatment, $n(\%)$ & $199(82.3)$ & $144(85.7)$ & $55(76.4)$ \\
\hline Need HCV medical support when receiving treatment, mean (SD) & $7.1(2.9)$ & $7.2(2.8)$ & $6.7(3.2)$ \\
\hline \multicolumn{4}{|l|}{ Barriers preventing patients from using these services } \\
\hline Worry that others know about the infection, mean (SD) & $3.6(3.6)$ & $3.8(3.6)$ & $3.1(3.6)$ \\
\hline Not engaging in risky behavior, will not suffer from HCV, mean (SD) & $2.6(3.3)$ & $2.3(3.1)$ & $3.4(3.8)$ \\
\hline No physical discomfort, do not need to treat HCV infection, mean (SD) & $3.2(3.5)$ & $2.8(3.3)$ & $4.2(3.7)$ \\
\hline Worry about withdrawal is unsanitary, so do not test, mean (SD) & $2.6(3.1)$ & 2.19 (2.98) & $2.73(3.52)$ \\
\hline Cannot afford treatment, $n(\%)$ & $184(77.3)$ & $133(79.2)$ & $51(72.3)$ \\
\hline Cannot find HCV treatment sites, $n(\%)$ & $132(55.2)$ & $98(58.3)$ & $34(47.9)$ \\
\hline Doctors cannot comply with the orders, adhere to long-term treatment, $n(\%)$ & $111(46.4)$ & $80(47.6)$ & $31(43.7)$ \\
\hline Do not have enough time, $n(\%)$ & $139(57.9)$ & $96(57.1)$ & $43(59.7)$ \\
\hline Satisfied with HCV testing service, mean (SD) & $7.7(2.7)$ & $7.5(2.7)$ & $8.1(2.7)$ \\
\hline Satisfied with HCV education in current treatment site, mean (SD) & $7.0(2.6)$ & $6.9(2.5)$ & $6.8(2.9)$ \\
\hline Satisfied with medical support, mean (SD) & $7.4(2.7)$ & $7.2(2.7)$ & $7.7(2.6)$ \\
\hline
\end{tabular}

counseling and video programs, respectively. More than $60 \%$ of staff reported providing pre- and post$\mathrm{HCV}$ test counseling in their routine work; however, only $31.1 \%$ reported providing further testing services, such as PCR or genotype testing. HCV-related medical service was sub-optimal: $45.6 \%$ of staff reported providing information on side effect management to patients and $30.5 \%$ provided referral services for $\mathrm{HCV}$-positive patients. Other medical treatment services were rare in their routine work. The situation of HCV-related services provided is summarized in Table 4.

\section{Discussion}

\section{Main findings of this study}

To the best of our knowledge, this study was the first to investigate $\mathrm{HCV}$ knowledge, related services provided, and barriers against $\mathrm{HCV}$-related services for both patients and staff in MMT clinics in China. In the current study, $70 \%$ of the participants were $\mathrm{HCV}$ positive; thus, the infection rate was significantly higher than in two other studies conducted in MMT clinics 3 years ago (57.0 and $51.3 \%$, respectively) $[7,16]$. Consistent with previous studies, many HCV-positive patients were not aware of their serostatus due to limited knowledge and the long duration of clinical progression of $\mathrm{HCV}$ infection. There were no epidemiological or longitudinal data; thus, we could not determine the reasons for the increasing $\mathrm{HCV}$ infection rate, but the sharp increase of infection rate shows us that long-term follow-up is necessary to monitor changes in patients' HCV status.
Regarding HCV knowledge, similar to other studies conducted in China and the western countries, both patients and staff had limited HCV knowledge. The patient HCV knowledge score was the same as a previous study in China [16], whereas it was lower than that reported in a US study, which had a mean score of 11.1 (4.1) [21]. The extremely poor HCV knowledge of the MMT staff (mean score of 10.9 (3.1)) is alarming because given individual's perceptions about drug-related risks are determined in part by their knowledge. The limited knowledge would be a greater barrier for patients accessing HCV-related services and can also decrease staff's self-confidence to provide related services. Similar to a previous study, needle sharing rates were fairly low compared to other regions in China $[7,8]$.

Unprotected sexual behavior was confirmed in this study; almost $10 \%$ of patients reported having had multiple sexual partners, and more than $10 \%$ reported not using condoms with casual or commercial partners. The primary route of $\mathrm{HCV}$ transmission was through infected blood; it remains controversial whether hepatitis $\mathrm{C}$ can be transmitted through sexual activity. However, condom use was also recommended to prevent $\mathrm{HCV}$ transmission, particularly in those with multiple partners who might have a higher risk of erosion to the genitalia. Because drug users are a high-risk population for HCV transmission, protective sexual behavior education should be provided in the prevention program.

Other risk factors that will potentially accelerate the progress to severe liver disease in $\mathrm{HCV}$-positive patients, 
Table 4 MMT staff characteristics and HCV services provided in their clinics

\begin{tabular}{ll}
\hline & Total \\
& $(n=59)$ \\
\hline Males, mean (SD) & $41(69.5)$ \\
Age, mean (SD) & $46.7(8.5)$ \\
Time of work in MMT clinics (months), mean (SD) & $45.3(20.5)$ \\
Education, $n$ (\%) & \\
College and higher & $14(24.1)$ \\
Less than college & $44(75.9)$ \\
Received HCV-related training & $10(16.9)$ \\
HCV knowledge scores & $10.9(3.1)$ \\
HCV education provided in their sites, $n$ (\%) & \\
Provided group counseling & $23(39.6)$ \\
Provided individual counseling & $36(62.1)$ \\
Provided TV/video program & $20(35.7)$ \\
Provided booklet & $34(58.6)$ \\
HCV testing provided in their sites, $n$ (\%) & \\
Pre-test counseling & $35(60.0)$ \\
Post-testing counseling & $41(69.5)$ \\
HCV antibody testing & $45(76.3)$ \\
Provided genotype/PCR testing & $18(31.1)$ \\
HCV medical treatment provided in their sites, $n(\%)$ & \\
Have psychical doctors & $8(13.5)$ \\
Provided referral service & $18(30.5)$ \\
\hline
\end{tabular}

including alcohol consumption and co-infection of $\mathrm{HCV}$ and HBV, have been identified in this study. These findings suggest that individual interventions should be provided to $\mathrm{HCV}$-positive patients in MMT clinics.

Barriers preventing patients from using HCV-related services As shown in Table 3, patients expressed the needs to receive $\mathrm{HCV}$-related education, testing, and medical support when they receive treatment, although there were no differences between $\mathrm{HCV}$-positive and HCV-negative patients. However, the patients also described their attitude regarding the barriers that may prevent them from using current services. More than three fourths of the patients reported that the cost of medical treatment prevented them from receiving medical treatment. Other barriers existed, such as difficulty in finding HCV treatment institutes and lacking time to receive treatment. In China, most drug users were not employed and thus did not have social health insurance. Therefore, even if they had the motivation to access medical treatment, economic barriers remained. These barriers prevented patients from accessing therapy, particularly the combination of multiple barriers in the system, which indicates that clinical interventions may not be enough to change the situation; therefore, the government should make more effective measures to improve access.

\section{HCV-related services in MMT treatment}

Overall, the results were not optimistic regarding the $\mathrm{HCV}$ knowledge of MMT staff and related services provided in their treatment site. A few more issues were present in this study. It is difficult for staff with limited education who are relatively older to learn new intervention skills. The average time spent working in MMT clinics was 45.3 (20.5) months; however, only $16.9 \%$ of staff reported received HCV-related training. Given the limited resources of these clinics, it is difficult for them to provide $\mathrm{HCV}$ education and counseling to patients during their routine work. More than half of the staff reported providing pre- and post-test counseling to patients, but most HCV-positive patients were not aware of their HCV status, which indicated that standard HCV testing counseling was not delivered to the patients. From our qualitative research, we found that there was no uniform procedure to inform patients of their $\mathrm{HCV}$ test results even though the HCV antibody test was a routine screening test in the MMT clinics. For example, a patient stated that "withdraw blood when I get the treatment, but nobody told me why", "staff did not tell me the test result, they posted it on the blackboard, I do not understand the meaning of positive and negative". Limited HCV service for drug users has been addressed in other studies as well. The barriers come from both individuals and the entire system and include the limited resources in MMT clinics, the overload of patient cases, and limited welfare for patients. $\mathrm{HCV}$ intervention should be a "combination intervention," that is, it should involve all possible resources; when all these services are combined in MMT clinics, the combination intervention will benefit the patients [22].

We acknowledge a number of limitations in this study. First, the patients in our study were recruited from Shanghai MMT clinics and thus may not be representative of patients in other areas. Second, we could not exclude the possibility of self-reported bias in the drug users. Finally, there may be other barriers or facilities that prevent or enhance the HCV service that we did not examine in the current study. Future work will be conducted to clarify these issues.

\section{Conclusions}

Our study indicated that $\mathrm{HCV}$ infection remains an important problem among MMT patients in China. 
Barriers for receiving $\mathrm{HCV}$-related services come from both the individual and the entire system. Effective intervention models must urgently be implemented to control $\mathrm{HCV}$ infection and associated consequences in China.

\section{Abbreviations}

AIDS: Acquired immune deficiency syndrome; AUDIT: The Alcohol Use Disorder Identification Test; BDI: Beck Depression Inventory; DSM-IV: Diagnostic and Statistical Manual IV; HCV: Hepatitis C virus; HIV: Human immunodeficiency virus; IDU: Injection drug use; MMT: Methadone maintenance treatment; NIDA: National Institute on Drug Abuse; RBS: Risk behaviors survey; SPSS: Statistical Package for the Social Science; WHO: World Health Organization

\section{Acknowledgements}

This study was conducted in Yangpu, Hongkou, Xuhui, and Minhang Mental Health Centers in Shanghai, China. The authors thank all the participants and staff for their collaboration. This work was supported by Shanghai Municipal Education Commission-Gaofeng Clinical Medicine Grant Support (20152235): $\mathrm{NIH}$ (1R01DA027195-01A2).

\section{Funding}

This study was supported by grants from the Shanghai Municipal Education Commission-Gaofeng Clinical Medicine Grant Support (20152235) and the National Institutes of Health (1R01DA027195). The study sponsors had no involvement in the study design, collection, analysis and interpretation of the data, writing of the manuscript, and decisions regarding publication.

\section{Availability of data and materials}

Please contact the author for data requests.

\section{Authors' contributions}

ZL conducted the data analysis and drafted the manuscript. LZ performed the statistical analysis and drafted the manuscript. JW participated in recruiting participants and collecting data in Yangpu. LH participated in recruiting participants and collecting data in Hongkou. ZZ participated in recruiting participants and collecting data in Xuhui. YC participated in recruiting participants and collecting data in Minhang. MZ provided suggestions and advice as a consultant. JD designed the study and revised the manuscript. All authors read and approved the final manuscript.

\section{Authors' information}

Biographical notes of the first authors: Zhi-Bin LI, female, born in 1986, M.D., majoring in drug abuse treatment. Lei ZHANG, female, born in 1991, Master of Psychology, majoring in Social Psychology and Mental Health.

\section{Ethics approval and consent to participate}

The research protocol was approved by the Ethics Committee of the Shanghai Mental Health Center, and each subject signed the informed consent form approved by the IRB at the Shanghai Mental Health Center. Board Name: IRB00002733-SHANGHAI MENTAL HEALTH CTR 2RB. The committee's reference number was NO. 2012-56C2.

\section{Consent for publication}

All participants signed consent forms.

\section{Competing interests}

The authors declare that they have no competing interests.

\section{Publisher's Note}

Springer Nature remains neutral with regard to jurisdictional claims in published maps and institutional affiliations.

\section{Author details}

'Shanghai Mental Health Center, Shanghai Jiao Tong University School of Medicine, Shanghai, China. ${ }^{2}$ Yangpu Mental Health Center, Shanghai, China. ${ }^{3}$ Hongkou Mental Health Center, Shanghai, China. ${ }^{4}$ Xuhui Mental Health Center, Shanghai, China. ${ }^{5}$ Minhang Mental Health Center, Shanghai, China. ${ }^{6}$ Jiading Mental Health Center, Shanghai, China. ${ }^{7}$ Shanghai Key Laboratory of
Psychotic Disorders, Shanghai, China. ${ }^{8}$ Brain Science and Technology Research Center, Shanghai Jiao Tong University, Shanghai, China.

Received: 1 August 2017 Accepted: 16 October 2017

Published online: 02 November 2017

References

1. World Health Organization. Guidelines for the screening, care and treatment of persons with hepatitis c infection. World Health Organ. 2014;172:343-6.

2. Chen YD, Liu MY, Yu WL, et al. Hepatitis $C$ virus infections and genotypes. HBPD Int. 2002:1:194-201.

3. China National Narcotics Control Commission. China drug report 2015. Beijing: NNCC; 2015

4. Walley AY, White MC, Kushel MB, Song YS, Tulsky JP. Knowledge of an interest in hepatitis $\mathrm{C}$ treatment at a methadone clinic. J Subst Abus Treat. 2005;28(2):181-7.

5. Doab A, Treloar C, Dore GJ. Knowledge and attitudes about treatment for hepatitis $C$ virus infection and barriers to treatment among current injection drug users in Australia. Clin Infect Dis. 2005;40(Suppl 5):S313-20.

6. Stoove MA, Gifford SM, Dore GJ. The impact of injecting drug use status on hepatitis C-related referral and treatment. Drug Alcohol Depend. 2005;77(1):81-6.

7. Hser Y, Du J, Li J, et al. Hepatitis $C$ among methadone maintenance treatment patients in Shanghai and Kunming, China. J Public Health (Oxf). 2012;34(1):24-31.

8. Du J, Wang Z, Xie B, et al. Hepatitis C knowledge and alcohol consumption among patients receiving methadone maintenance treatment in Shanghai, China. Am J Drug Alcohol Abuse. 2012;38(3):228-32.

9. Liang YX. HIV and HCV prevalence among entrants to methadone maintenance treatment clinics in china: a meta-analysis. Nantong: Nantong univ; 2013.

10. Wu ZY, Luo W, Sullivan S, et al. Evaluation of a needle social marketing strategy to control HIV among injecting drug users in China. AIDS. 2007; 21(Suppl. 8):S115-22.

11. Qian HZ, Joseph ES, Hury TC, et al. Injection drug use and HIV/AIDS in China: review of current situation, prevention and policy implication. Harm Reduct J. 2006:3(4):28-42.

12. Darke $\mathrm{S}, \mathrm{Hall}$ W, Heather $\mathrm{N}$, et al. The reliability and validity of a scale to measure HIV risk taking behavior among intravenous drug users. AIDS. 1991;5:181-5.21

13. Weatherby NL, Needle R, Cesari $H$, et al. Validity of self-reported drug use among injection drug users and crack cocaine users recruited through street outreach. Eval Program Plann. 1994;17:347-55.22.

14. Needle R, Fisher DG, Weatherby N, et al. Reliability of self-reported HIV risk behaviors of drug users. Psychol Addict Behav. 1995;9(4):242-50.

15. Strauss SM, Astone-Twerell JM, Munoz-Plaza C, et al. Hepatitis C knowledge among staff in U.S. drug treatment programs. J Drug Educ. 2006;36(2):141-58.

16. Jiang DU, Zhen $\mathrm{W}$, Zhang $\mathrm{H}$, et al. HCV knowledge and HCV infection among drug users treated in a methadone maintenance treatment clinic. Chin J Drug Depend. 2009:18(6):495-9.

17. Chang YJ, Hsieh J, Peng CY, et al. HIV and HCV serostatus and knowledge among patients in urban versus rural methadone maintenance clinics in Kunming. J Drug Issues. 2014;44(3):281-90.

18. World Health Organization. The alcohol use disorders identification test. Guidelines for use in primary care. Geneva: World Health Organization; 2001

19. Li B, Shen YC, Zhang BQ, et al. The test of audit in China. Chin Ment Health J. 2003:17(1):22-4

20. Wang $X D$, Wang $X L, M a H$, et al. Handbook of mental health assessment scale (Revised Edition) (1999). Beijing: Chinese Mental Health Journal; 2009.

21. Strauss S M, Astone-Twerell J, Munoz-Plaza C E, et al. (2007) Drug treatment program patients' hepatitis $\mathrm{C}$ virus (HCV) education needs and their use of available HCV education services [J] BMC Health Serv Res. 7:39.

22. Birkhead GS, Klein SJ. Integrating multiple programmer and policy approaches to hepatitis $C$ prevention and care for injection drug users: a comprehensive approach. Int J Drug Policy. 2007;18:417-25. 